\title{
Fixed point theorems for cyclic contraction mappings in fuzzy metric spaces
}

\author{
Yonghong Shen ${ }^{1,2^{*}}$ and Wei Chen ${ }^{3}$
}

\author{
"Correspondence: \\ shenyonghong2008@hotmail.com \\ 'School of Mathematics and \\ Statistics, Tianshui Normal \\ University, Tianshui, 741001, \\ P.R. China \\ ${ }^{2}$ School of Mathematics, Beijing \\ Institute of Technology, Beijing, \\ 100081, P.R. China \\ Full list of author information is \\ available at the end of the article
}

\begin{abstract}
In the present paper, an extension of the Edelstein contraction theorem for cyclic contractions in a fuzzy metric space is established, which also can be considered as a generalization of the fuzzy Edelstein contraction theorem introduced by Grabiec. Additionally, we extend a fixed point theorem in G-complete fuzzy metric spaces given by Shen et al. to $M$-complete fuzzy metric spaces. Meantime, two examples are constructed to illustrate the corresponding results, respectively.
\end{abstract}

\section{Introduction}

The contraction type mappings in fuzzy metric spaces play a crucial role in fixed point theory. In 1988, Grabiec [1] first defined the Banach contraction in a fuzzy metric space and extended fixed point theorems of Banach and Edelstein to fuzzy metric spaces. Following Grabiec's approach, Mishra et al. [2] obtained some common fixed point theorems for asymptotically commuting mappings in fuzzy metric spaces. In the sequel, Vasuki [3] offered a generalization of Grabiec's fuzzy Banach contraction theorem and proved a common fixed point theorem for a sequence of mappings in a fuzzy metric space. Afterwards, Cho [4] presented the concept of compatible mappings of type $(\alpha)$ in fuzzy metric spaces and then studied the fixed point theory. Several years later, Singh and Chauhan [5] introduced the concept of compatible mapping and proved two common fixed point theorems in the fuzzy metric space with the strongest triangular norm. In 2002, Sharma [6] further extended some known results of fixed point theory for compatible mappings in fuzzy metric spaces. In the same year, Gregori and Sapena [7] introduced the notion of fuzzy contractive mapping and presented some fixed point theorems for complete fuzzy metric spaces in the sense of George and Veeramani, and also for Kramosil and Michalek's fuzzy metric spaces which are complete in Grabiec's sense. Soon after, Mihet [8] proposed a fuzzy Banach theorem for (weak) $B$-contraction in $M$-complete fuzzy metric spaces. Later, Mihet $[9,10]$ further studied the fixed point theory for the different contraction mappings in fuzzy metric spaces, and introduced some new contraction mappings, such as Edelstein fuzzy contractive mappings, fuzzy $\psi$-contraction of $(\varepsilon, \lambda)$ type, etc. Based on the definition of fuzzy contractive mapping introduced by Mihet [9], Abbas et al. [11] proposed the notion of $\varphi$-weak contraction and obtained several results of fixed point in a $G$-complete metric space. Recently, Shen et al. [12] constructed a novel class of $\varphi$-contractions and proved a fixed point theorem for this kind of mappings in an $M$-complete fuzzy metric space.

C) 2013 Shen and Chen; licensee Springer. This is an Open Access article distributed under the terms of the Creative Commons Attribution License (http://creativecommons.org/licenses/by/2.0), which permits unrestricted use, distribution, and reproduction in any medium, provided the original work is properly cited. 
In 2003, Kirk et al. [13] first introduced a novel class of contractive conditions, namely, cyclical contractive conditions, and extended several fixed point theorems including Banach, Edelsetin, Carsti and a fixed point theorem for nonexpansive mappings in a Banach space. Motivated by the notion of cyclic contraction, Păcurar and Ioan [14] proposed the concept of cyclic $\varphi$-contraction. Based on this type of contractive condition, they constructed a fixed point theorem in a classical complete metric space. Moreover, several problems related to the fixed point are investigated, such as data dependence, well-posedness, limit shadowing property, and so on. Hereafter, Karapinar [15] proposed the concept of cyclic weak $\phi$-contraction, and then discussed some main results proposed by Păcurar and Ioan [14] under this kind of contractive conditions. Following these ideas mentioned above, Shen et al. [16] extended the notion of cyclic $\varphi$-contraction to fuzzy metric spaces, and proposed a fixed point theorem for this type of mappings in G-complete fuzzy metric spaces in the sense of George and Veeramani. Simultaneously, some of the results concerned with the fixed point proposed by Păcurar and Ioan [14] were also generalized to fuzzy metric spaces. Analogously, Gopal et al. [17] also introduced the notion of cyclic weak $\varphi$-contraction in fuzzy metric spaces and obtained some results on the existence and uniqueness of a fixed point in fuzzy metric spaces in the sense of G-completeness. Furthermore, Murthy et al. [18] generalized the concept of cyclic weak $\varphi$-contraction to cyclic weak $(\varphi, \psi)$-contraction and utilized these contractive mappings to prove a fixed point theorem in a $G$-complete fuzzy metric space.

In this paper, we present an extension of the Edelstein contraction theorem for cyclic contractions in a fuzzy metric space. Our results can also be viewed as a generalization of the fuzzy Edelstein contraction theorem proposed by Grabiec [1]. Besides, we also generalized a fixed theorem in G-complete fuzzy metric spaces given by Shen et al. [16] to $M$-complete fuzzy metric spaces. In addition, it should be pointed out that the function $\varphi$ of cyclic $\varphi$-contraction proposed by us is different from that of cyclic $\varphi$-contraction introduced in $[17,18]$.

\section{Preliminaries}

For completeness and clarity, in this section, some related concepts and conclusions are summarized and introduced below. Let $\mathbb{N}$ denote the set of all positive integers.

Definition 2.1 (Schweizer and Sklar [19]) A binary operation $*:[0,1] \times[0,1] \rightarrow[0,1]$ is called a continuous triangular norm (in short, continuous $t$-norm) if it satisfies the following conditions:

$(\mathrm{TN}-1) *$ is commutative and associative;

(TN-2) $*$ is continuous;

(TN-3) $a * 1=a$ for every $a \in[0,1]$

(TN-4) $a * b \leq c * d$ whenever $a \leq c, b \leq d$ and $a, b, c, d \in[0,1]$.

Definition 2.2 (George and Veeramani [20]) A fuzzy metric space is an ordered triple $(X, M, *)$ such that $X$ is a nonempty set, $*$ is a continuous $t$-norm and $M$ is a fuzzy set on $X \times X \times(0, \infty)$ satisfying the following conditions for all $x, y, z \in X, s, t>0$ :

$(\mathrm{FM}-1) M(x, y, t)>0$

(FM-2) $M(x, y, t)=1$ if and only if $x=y$;

$(\mathrm{FM}-3) M(x, y, t)=M(y, x, t)$; 
$(\mathrm{FM}-4) \quad M(x, y, t) * M(y, z, s) \leq M(x, z, t+s)$

$(\mathrm{FM}-5) M(x, y, \cdot):(0, \infty) \rightarrow(0,1]$ is continuous.

Definition 2.3 (George and Veeramani [20]) Let $(X, M, *)$ be a fuzzy metric space. Then:

(i) A sequence $\left\{x_{n}\right\}$ in $X$ is said to converge to $x$ in $X$, denoted by $\lim _{n \rightarrow \infty} x_{n}=x$ (or $\left.x_{n} \rightarrow x\right)$, if and only if $\lim _{n \rightarrow \infty} M\left(x_{n}, x, t\right)=1$ for all $t>0$, i.e., for each $r \in(0,1)$ and $t>0$, there exists $n_{0} \in \mathbb{N}$ such that $M\left(x_{n}, x, t\right)>1-r$ for all $n \geq n_{0}$;

(ii) A sequence $\left\{x_{n}\right\}$ is an $M$-Cauchy sequence if and only if for all $\epsilon>0$, there exists $n_{0} \in \mathbb{N}$ such that $M\left(x_{m}, x_{n}, t\right)>1-\epsilon$ for any $m, n>n_{0}$ and $t>0$;

(iii) A fuzzy metric space $(X, M, *)$ is called $M$-complete if every $M$-Cauchy sequence is convergent.

Notice that a fuzzy metric space is called compact if every sequence contains a convergent subsequence.

Lemma 2.1 (Grabiec [1]) Let $\lim _{n \rightarrow \infty} x_{n}=x$ and $\lim _{n \rightarrow \infty} y_{n}=y$. If $M(x, y, \cdot)$ is continuous, then $\lim _{n \rightarrow \infty} M\left(x_{n}, y_{n}, t\right)=M(x, y, t)$ for any $t>0$.

Lemma 2.2 (Grabiec [1], fuzzy Edelstein contraction theorem) Let $(X, M, *)$ be a compact fuzzy metric space. $T: X \rightarrow X$ a mapping satisfying the following condition:

$M(T x, T y, \cdot)>M(x, y, \cdot)$

for all $x \neq y$. Then $T$ has a unique fixed point.

Definition 2.4 (Păcurar and Rus [14]) Let $X$ be a nonempty set, $r$ be a positive integer and $f: X \rightarrow X$ be a mapping. $X=\bigcup_{i=1}^{r} X_{i}$ is a cyclic representation of $X$ with respect to $f$ if

(i) $X_{i}, i=1,2, \ldots, r$, are nonempty sets;

(ii) $f\left(X_{1}\right) \subset X_{2}, \ldots, f\left(X_{r-1}\right) \subset X_{r}, f\left(X_{r}\right) \subset X_{1}$.

Definition 2.5 A function $\varphi:[0,1] \rightarrow[0,1]$ is called a comparison function if it satisfies:

(i) $\varphi$ is nondecreasing and left continuous;

(ii) $\varphi(t)>t$ for all $t \in(0,1)$.

Lemma 2.3 (Vetro [21]) If $\varphi$ is a comparison function, then

(i) $\varphi(1)=1$;

(ii) $\lim _{n \rightarrow+\infty} \varphi^{n}(t)=1$ for all $t \in(0,1)$, where $\varphi^{n}(t)$ denotes the composition of $\varphi(t)$ with itself $n$ times.

Definition 2.6 (Shen et al. [16]) Let $(X, M, *)$ be a fuzzy metric space, $r$ be a positive integer, $A_{1}, A_{2}, \ldots, A_{r} \in P_{\mathrm{cl}}(X)$, where $P_{\mathrm{cl}}(X)$ denotes the collection of nonempty closed subsets of $X, Y=\bigcup_{i=1}^{r} A_{i}$ and $f: Y \rightarrow Y$ a mapping. If

(i) $\bigcup_{i=1}^{r} A_{i}$ is cyclic representation of $Y$ with respect to $f$;

(ii) there exists a comparison function $\varphi:[0,1] \rightarrow[0,1]$ such that

$$
M(f(x), f(y), t) \geq \varphi(M(x, y, t))
$$


for any $x \in A_{i}, y \in A_{i+1}$ and $t>0$, where $A_{r+1}=A_{1}$, then $f$ is called cyclic $\varphi$-contraction in the fuzzy metric space.

\section{Main results}

In this section, we prove an extended fuzzy Edelstein contraction theorem for cyclic contractive mappings in a fuzzy metric space. Meantime, we also prove that a fixed point theorem given by Shen et al. [16] does hold even if G-completeness of the fuzzy metric space is replaced by $M$-completeness.

Theorem 3.1 (The extended fuzzy Edelstein contraction theorem) Let $(X, M, *)$ be a fuzzy metric space, $r$ be a positive integer, $A_{1}, A_{2}, \ldots, A_{r} \in P_{\mathrm{cl}}(X)$, at least one of which is compact. $Y=\bigcup_{i=1}^{r} A_{i}, f: Y \rightarrow Y$ a mapping. Assume that

(C1) $\bigcup_{i=1}^{r} A_{i}$ is a cyclic representation of $Y$ with respect to $f$;

(C2) $M(f(x), f(y), \cdot)>M(x, y, \cdot)$ for any $x \in A_{i}, y \in A_{i+1}(x \neq y), i=1,2, \ldots, r$.

Then $f$ has a unique fixed point $x^{*} \in \bigcap_{i=1}^{r} A_{i}$.

Proof Without loss of generality, we assume that $A_{1}$ is compact. Define $d(t)=\sup \{M(x$, $\left.y, t): x \in A_{1}, y \in A_{r}\right\}$ for each $t>0$. According to Definition 2.2, we know that $0<d(t) \leq 1$. Since $A_{1}$ is compact, for an arbitrary but fixed $t>0$, there exist $x_{0} \in A_{1}$ and a sequence $\left\{y_{n}\right\} \subset A_{r}$ such that $\lim _{n \rightarrow \infty} M\left(x_{0}, y_{n}, t\right)=d(t)$. Now, we claim that $d(t)=1$ for each given $t>0$. Otherwise, we can assume that $0<d(t)<1$. By the condition (FM-2), we know that $x_{0} \neq y_{n}$ for each $n \in \mathbb{N}$. Furthermore, we can obtain that $f^{i}\left(x_{0}\right) \neq f^{i}\left(y_{n}\right)$ for $1 \leq i \leq r$. Thus, for every $n \in \mathbb{N}$, we have

$$
\begin{aligned}
M\left(f^{r+1}\left(x_{0}\right), f^{r+1}\left(y_{n}\right), t\right) & >M\left(f^{r}\left(x_{0}\right), f^{r}\left(y_{n}\right), t\right) \\
& >\cdots>M\left(f\left(x_{0}\right), f\left(y_{n}\right), t\right)>M\left(x_{0}, y_{n}, t\right) .
\end{aligned}
$$

Since the sequence $\left\{f^{r+1}\left(y_{n}\right)\right\} \subset A_{1}$ and the compactness of $A_{1}$, there exists a subsequence of $\left\{f^{r+1}\left(y_{n}\right)\right\}$ such that it converges to some $z \in A_{1}$. According to the foregoing inequality and Lemma 2.1, by supposing that $n \rightarrow \infty$, it follows that

$$
M\left(f^{r+1}\left(x_{0}\right), z, t\right) \geq d(t) .
$$

According to the arbitrariness of $t$, if $M\left(f^{r+1}\left(x_{0}\right), z, t\right)=1$, then we have $f^{r+1}\left(x_{0}\right)=z$. Furthermore, we can obtain that $M\left(f^{2 r}\left(x_{0}\right), f^{r-1}(z), t\right)=1$. Obviously, this is a contradiction. On the other hand, if $M\left(f^{r+1}\left(x_{0}\right), z, t\right)<1$, then we have $f^{r+1}\left(x_{0}\right) \neq z$. Thus, by the conditions $(\mathrm{C} 1)$ and $(\mathrm{C} 2)$, we can obtain

$$
\begin{aligned}
M\left(f^{2 r}\left(x_{0}\right), f^{r-1}(z), t\right) & >M\left(f^{2 r-1}\left(x_{0}\right), f^{2 r-2}(z), t\right) \\
& >\cdots>M\left(f^{r+1}\left(x_{0}\right), z, t\right) \geq d(t) .
\end{aligned}
$$

As $f^{2 r}\left(x_{0}\right) \in A_{1}$ and $f^{r-1}(z) \in A_{r}$, this implies a contradiction. Therefore, we conclude that $d(t)=1$ for each $t>0$, and $A_{1} \cap A_{r} \neq \emptyset$. By $(\mathrm{C} 1), f\left(A_{1} \cap A_{r}\right) \subset A_{1}$ and $f\left(A_{1} \cap A_{r}\right) \subset A_{2}$, we have $f\left(A_{1} \cap A_{r}\right) \subset A_{1} \cap A_{2}$. Obviously, $A_{1} \cap A_{2} \neq \emptyset$.

Now, we redefine the sets $A_{1}^{\prime}=A_{1} \cap A_{2}, A_{2}^{\prime}=A_{2} \cap A_{3}, \ldots, A_{r}^{\prime}=A_{r} \cap A_{1}$. In view of (C1), these sets are both nonempty and closed. As $A_{1}^{\prime}$ is compact, the conditions (C1) and (C2) 
are satisfied by $f$ with respect to the family $\left\{A_{i}^{\prime}\right\}, i=1,2, \ldots, r$. By repeating the above process, we obtain $A_{1}^{\prime} \cap A_{r}^{\prime} \neq \emptyset$, which implies $A_{1} \cap A_{2} \cap A_{3} \neq \emptyset$.

Continuing in this manner, we can obtain

$$
A:=\bigcap_{i=1}^{r} A_{i} \neq \emptyset
$$

Consider the restriction $\left.f\right|_{A}$ of $f$ on $A$. Obviously, the mapping $\left.f\right|_{A}: A \rightarrow A$ satisfies the condition (C2) and $A$ is compact. By Lemma 2.2, we conclude that $\left.f\right|_{A}$ has a unique fixed point in $A$. However, by the condition (C1), it can easily be shown that the uniqueness follows from the fact that any fixed point of $f$ necessarily lies in $A$.

Remark 1 Theorem 3.1 can be regarded as an extension of the fuzzy Edelstein contraction theorem (Lemma 2.2) given by Grabiec [1], and it is not related with the compactness and completeness of the fuzzy metric space $(X, M, *)$.

Example 1 Let $X=[0,4]$ be endowed with the usual metric $d(x, y)=|x-y|$. Define $M(x, y, t)=\frac{t}{t+|x-y|}$ for all $x, y \in X$ and $t>0$. Clearly, $(X, M, *)$ is a fuzzy metric space with respect to $t$-norm $a * b=a b$.

Let $f: X \rightarrow X$ be defined as $f(x)=2-\frac{x}{2}$. Set $A_{1}=[0,2], A_{2}=[1,4]$. Owing to $f\left(A_{1}\right)=$ $[1,2] \subset[1,4]=A_{2}, f\left(A_{2}\right)=\left[0, \frac{3}{2}\right] \subset[0,2]=A_{1}$, we can obtain that $A_{1} \cup A_{2}$ is a cyclic representation of $X$ with respect to $f$. In addition, for any $x \in A_{1}, y \in A_{2}$ and $x \neq y$, we have

$$
M(f(x), f(y), t)=\frac{t}{t+\frac{1}{2}|x-y|}>\frac{t}{t+|x-y|}=M(x, y, t) .
$$

Thus, all the conditions of Theorem 3.1 are satisfied and $x=\frac{4}{3}$ is the unique fixed point of $f$.

Theorem 3.2 Let $(X, M, *)$ be an $M$-complete fuzzy metric space, $r$ be a positive integer, $A_{1}, A_{2}, \ldots, A_{r} \in P_{\mathrm{cl}}(X), Y=\bigcup_{i=1}^{r} A_{i}, \varphi:[0,1] \rightarrow[0,1]$ be a comparison function, and $f: Y \rightarrow$ $Y$ be a mapping. Assume that

(C1) $\bigcup_{i=1}^{r} A_{i}$ is a cyclic representation of $Y$ with respect to $f$;

(C2) $f$ is a cyclic $\varphi$-contraction.

Then $f$ has a unique fixed point $x^{*} \in \bigcap_{i=1}^{r} A_{i}$ and the iterative sequence $\left\{x_{n}\right\}_{n \geq 0}\left(x_{n}=\right.$ $\left.f\left(x_{n-1}\right), n \in \mathbb{N}\right)$ converges to $x^{*}$ for any initial point $x_{0} \in Y$.

Proof Let $x_{0} \in Y$ be an arbitrary initial point. Define $\tau_{n}(t)=M\left(x_{n}, x_{n+1}, t\right)$ for all $n \in \mathbb{N} \cup\{0\}$, $t>0$. Now, we show that $f$ has a fixed point. In particular, if there exists an $n_{0} \in \mathbb{N} \cup$ $\{0\}$ such that $x_{n_{0}+1}=x_{n_{0}}$, i.e., $f\left(x_{n_{0}}\right)=x_{n_{0}}$, then it is obvious that $x_{n_{0}}$ is a fixed point of $f$. Otherwise, the inequality $x_{n} \neq x_{n+1}$ holds for each $n$, which implies that $0<\tau_{n}(t)<1$ for all $n \in \mathbb{N} \cup\{0\}$ and $t>0$. By $(\mathrm{C} 2),\left\{\tau_{n}(t)\right\}$ is monotone increasing and bounded from below for every $t>0$ with respect to $n$. So, $\lim _{n \rightarrow \infty} \tau_{n}(t)=\tau(t) \leq 1$. Suppose that $0<\tau(t)<1$. Since

$$
\tau_{n+1}(t)=M\left(x_{n+1}, x_{n+2}, t\right) \geq \varphi\left(M\left(x_{n}, x_{n+1}, t\right)\right)=\varphi\left(\tau_{n}(t)\right)
$$

for each $t$, letting $n \rightarrow \infty$, we conclude that $\tau(t) \geq \varphi(\tau(t))$. Obviously, which is a contradiction. Thus, we obtain $\lim _{n \rightarrow \infty} \tau_{n}(t)=1$. 
Next, we show that the iterative sequence $\left\{x_{n}\right\}_{n \geq 0}$ is an $M$-Cauchy sequence. Suppose that it is not. Then there exist $\epsilon \in(0,1), t>0$, and two sequences $\{p(n)\}$ and $\{q(n)\}$ such that for every $n \in \mathbb{N} \cup\{0\}$, so we obtain that

$$
p(n)>q(n) \geq n, \quad M\left(x_{p(n)}, x_{q(n)}, t\right) \leq 1-\epsilon .
$$

Furthermore, for each $n$, we can choose the smallest number $p(n)$ greater than $q(n)$ such that

$$
M\left(x_{p(n)-1}, x_{q(n)-1}, t\right)>1-\epsilon, \quad M\left(x_{p(n)-1}, x_{q(n)}, t\right)>1-\epsilon .
$$

For each $n \in \mathbb{N} \cup\{0\}$, we put $s_{n}(t)=M\left(x_{p(n)}, x_{q(n)}, t\right)$. Then we have

$$
\begin{aligned}
1-\epsilon \geq s_{n}(t) & =M\left(x_{p(n)}, x_{q(n)}, t\right) \\
& \geq M\left(x_{p(n)-1}, x_{p(n)}, t / 2\right) * M\left(x_{p(n)-1}, x_{q(n)}, t / 2\right) \\
& \geq \tau_{p(n)-1}(t / 2) *(1-\epsilon) .
\end{aligned}
$$

Since $\tau_{p(n)-1}(t / 2) \rightarrow 1$ as $n \rightarrow \infty$ for the foregoing $t$, letting $n \rightarrow \infty$, we conclude that the sequence $\left\{s_{n}(t)\right\}$ converges to $1-\epsilon$. Moreover, for $p(n)>q(n)$, there exists $0 \leq j \leq r-1$ such that $p(n)-q(n)+j=1 \bmod r$ for infinitely many $n$.

Case I: If $j=0$, then, for such $n$, we have

$$
\begin{aligned}
s_{n}(t) & =M\left(x_{p(n)}, x_{q(n)}, t\right) \\
& \geq M\left(x_{p(n)}, x_{p(n)+1}, t / 3\right) * M\left(x_{p(n)+1}, x_{q(n)+1}, t / 3\right) * M\left(x_{q(n)+1}, x_{q(n)}, t / 3\right) \\
& \geq \tau_{p(n)}(t / 3) * \varphi\left(M\left(x_{p(n)}, x_{q(n)}, t / 3\right)\right) * \tau_{q(n)}(t / 3) \\
& =\tau_{p(n)}(t / 3) * \varphi\left(s_{n}(t)\right) * \tau_{q(n)}(t / 3) .
\end{aligned}
$$

Letting $n \rightarrow \infty$, we obtain that

$$
1-\epsilon \geq \varphi(1-\epsilon)>1-\epsilon \text {. }
$$

Clearly, this leads to a contradiction.

Case II: If $j \neq 0$, similarly, for such $n$, we can obtain

$$
\begin{aligned}
s_{n}(t)= & M\left(x_{p(n)}, x_{q(n)}, t\right) \\
\geq & M\left(x_{p(n)}, x_{p(n)+1}, t /(j+3)\right) * M\left(x_{p(n)+1}, x_{p(n)+2}, t /(j+3)\right) * \cdots \\
& * M\left(x_{p(n)+j}, x_{p(n)+j+1}, t /(j+3)\right) * M\left(x_{p(n)+j+1}, x_{q(n)+1}, t /(j+3)\right) \\
& * M\left(x_{q(n)+1}, x_{q(n)}, t /(j+3)\right) \\
\geq & \tau_{p(n)}(t /(j+3)) * \tau_{p(n)+1}(t /(j+3)) * \cdots * \tau_{p(n)+j}(t /(j+3)) \\
& * \varphi\left(M\left(x_{p(n)+j}, x_{q(n)}, t /(j+3)\right)\right) * \tau_{q(n)}(t /(j+3)) .
\end{aligned}
$$

Supposing that $n \rightarrow \infty$, we can get the same contradiction as Case I. 
Moreover, we consider another case. That is, there exists $n_{0} \in \mathbb{N} \cup\{0\}$ such that $M\left(x_{m}, x_{n}, t\right) \leq 1-\epsilon$ for all $m, n \geq n_{0}$. Therefore, for any $p \in \mathbb{N}$, it is obvious that $M\left(x_{n_{0}+p+1}, x_{n_{0}+p+2}, t\right) \leq 1-\epsilon$. By $(\mathrm{C} 2)$, we know that the sequence $\left\{M\left(x_{n_{0}+p+1}, x_{n_{0}+p+2}, t\right)\right\}$ is monotone increasing and bounded with respect to $p$ for each $t$. So, there exists $\alpha(t) \in(0,1-\epsilon]$ such that $\lim _{p \rightarrow \infty} M\left(x_{n_{0}+p+1}, x_{n_{0}+p+2}, t\right)=\alpha(t)$. Thus, we can obtain

$$
M\left(x_{n_{0}+p+2}, x_{n_{0}+p+3}, t\right) \geq \varphi\left(M\left(x_{n_{0}+p+1}, x_{n_{0}+p+2}, t\right)\right) .
$$

Letting $p \rightarrow \infty$, for the forgoing $t$, we have $\alpha(t) \geq \varphi(\alpha(t))$, which is also a contradiction.

Based on the above analysis, we obtain that $\left\{x_{n}\right\}_{n \geq 0}$ is an $M$-Cauchy sequence in the $M$-complete fuzzy metric subspace $Y$. Furthermore, we conclude that there exists an $x^{*} \in$ $Y$ such that $\lim _{n \rightarrow \infty} x_{n}=x^{*}$.

Now, we show that $x^{*}$ is a fixed point of $f$. By the condition (C1), it follows that the iterative sequence $\left\{x_{n}\right\}_{n \geq 0}$ has an infinite number of terms in each $A_{i}, i=1,2, \ldots, r$. Since $Y$ is $M$-complete, from each $A_{i}, i=1,2, \ldots, r$, we can extract a subsequence of $\left\{x_{n}\right\}_{n \geq 0}$ which converges to $x^{*}$ as well. Because each $A_{i}, i=1,2, \ldots, r$, is closed, we conclude that $x^{*} \in \bigcap_{i=1}^{r} A_{i}$ and thus $\bigcap_{i=1}^{r} A_{i} \neq \emptyset$.

Set $Z=\bigcap_{i=1}^{r} A_{i}$. Clearly, $Z$ is closed and $M$-complete. Consider the restriction of $f$ on $Z$, that is, $\left.f\right|_{Z}: Z \rightarrow Z$. Next, we show that $\left.f\right|_{Z}$ has a unique fixed point in $Z \subset Y$.

For the forgoing $x^{*} \in Z$, there exists $i_{n}(n \in \mathbb{N})$ such that $x_{n} \in A_{i_{n}}$. Therefore, we can choose $A_{i_{n}+1}$ such that $\left.f\right|_{Z}\left(x^{*}\right) \in A_{i_{n}+1}$. Accordingly, for any $t>0$, we can obtain

$$
\begin{aligned}
M\left(\left.f\right|_{Z}\left(x^{*}\right), x^{*}, t\right) & =M\left(f\left(x^{*}\right), x^{*}, t\right) \\
& \geq M\left(f\left(x^{*}\right), f\left(x_{n}\right), t / 2\right) * M\left(x_{n+1}, x^{*}, t / 2\right) \\
& \geq \varphi\left(M\left(x^{*}, x_{n}, t / 2\right)\right) * M\left(x_{n+1}, x^{*}, t / 2\right) .
\end{aligned}
$$

By supposing that $n \rightarrow \infty$, we conclude that $M\left(\left.f\right|_{Z}\left(x^{*}\right), x^{*}, t\right)=1$ for any $t>0$, i.e., $\left.f\right|_{Z}\left(x^{*}\right)=x^{*}$. That is to say, $x^{*}$ is a fixed point of $\left.f\right|_{Z}$, which is obtained by iteration from the initial point $x_{0} \in Y$.

To show uniqueness, we assume that $y \in \bigcap_{i=1}^{r} A_{i}$ is another fixed point of $\left.f\right|_{z}$. As $x^{*}, y \in$ $A_{i}$ for every $i \in\{1,2, \ldots, r\}$, we obtain that

$$
\begin{aligned}
M\left(x^{*}, y, t\right) & =M\left(\left.f\right|_{Z}\left(x^{*}\right),\left.f\right|_{Z}(y), t\right) \\
& =M\left(f\left(x^{*}\right), f(y), t\right) \\
& \geq \varphi\left(M\left(x^{*}, y, t\right)\right) \\
& >M\left(x^{*}, y, t\right) .
\end{aligned}
$$

This leads to a contradiction. Thus, $x^{*}$ is a unique fixed point of $\left.f\right|_{Z}$.

Finally, we have to prove that the iterative sequence $\left\{x_{n}\right\}_{n \geq 0}$ converges to $x^{*}$ for any starting point $x_{0} \in Y$. Since $x_{0} \in Y=\bigcup_{i=1}^{m} A_{i}$, there exists $i_{0} \in\{1,2, \ldots, r\}$ such that $x_{0} \in A_{i_{0}}$. As $x^{*} \in \bigcap_{i=1}^{m} A_{i}$, we know that $x^{*} \in A_{i_{0}+1}$ as well. Then, for any $t>0$, we have

$$
M\left(f(x), f\left(x^{*}\right), t\right) \geq \varphi\left(M\left(x, x^{*}, t\right)\right) .
$$


By induction, we can obtain

$$
\begin{aligned}
M\left(x_{n}, x^{*}, t\right) & =M\left(f^{n}\left(x_{0}\right), x^{*}, t\right) \\
& =M\left(f^{n}\left(x_{0}\right), f\left(x^{*}\right), t\right) \\
& =M\left(f\left(f^{n-1}\right)\left(x_{0}\right), f\left(x^{*}\right), t\right) \\
& \geq \varphi\left(M\left(f^{n-1}\left(x_{0}\right), x^{*}, t\right)\right) \\
& \geq \cdots \geq \varphi^{n}\left(M\left(x_{0}, x^{*}, t\right)\right) .
\end{aligned}
$$

According to Lemma 2.3, supposing $x_{0} \neq x^{*}$, it follows immediately that $x_{n} \rightarrow x^{*}$ as $n \rightarrow \infty$. Hence, the iterative sequence $\left\{x_{n}\right\}_{n \geq 0}$ converges to the unique fixed point $x^{*}$ of $f$ for any initial point $x_{0} \in Y$. This completes the proof of the theorem.

Remark 2 Based on Theorem 3.2, the remaining results concerning fixed point theory given in [16] can be directly obtained in the sense of $M$-completeness.

Example 2 Let $X=[0,3]$ be equipped with the ordinary metric $d(x, y)=|x-y|, \varphi(\tau)=\sqrt{\tau}$ for all $\tau \in[0,1]$. Define $M(x, y, t)=e^{-\frac{2|x-y|}{t}}$ for all $x, y \in X$ and $t>0$. Clearly, $(X, M, *)$ is an $M$-complete fuzzy metric space with respect to $t$-norm $a * b=a b$.

Let $f: X \rightarrow X$ be defined as

$$
f(x)= \begin{cases}1, & x \in[0,1] \\ \frac{3-x}{2}, & x \in(1,3] .\end{cases}
$$

Set $A_{1}=\left[\frac{2}{3}, 3\right], A_{2}=\left[0, \frac{3}{2}\right]$, it is obvious that $f\left(A_{1}\right)=[0,1] \subseteq A_{2}, f\left(A_{2}\right)=\left[\frac{3}{4}, 1\right] \subseteq A_{1}$. Besides, for any $x \in A_{1}, y \in A_{2}$, we have

$$
d(f(x), f(y))=|f(x)-f(y)| \leq \frac{1}{2}|x-y| \leq|x-y|=d(x, y),
$$

it follows that $M(f(x), f(y), t)=e^{-\frac{2|f(x)-f(y)|}{t}} \geq e^{-\frac{|x-y|}{t}}=\varphi(M(x, y, t))$ for every $t>0$. Thus, $f$ is a cyclic $\varphi$-contraction in the fuzzy metric space $(X, M, *)$. Now, all the conditions of Theorem 3.2 are satisfied and then $f$ has a unique fixed point, that is $x=1$.

\section{Conclusion}

In this paper, we have proposed an extended version of the Edelstein contraction theorem for cyclic contraction mappings in a fuzzy metric space, which can be considered as a generalization of the fuzzy Edelstein contraction theorem introduced by Grabiec [1]. It should be noted that the existence of a unique fixed point, in our results, does not depend on the compactness and completeness of a fuzzy metric space. Moreover, we have also generalized a fixed point theorem given by Shen et al. [16] to an $M$-complete fuzzy metric space. The conclusion has shown that the original proposition and the corresponding conclusions are true even if $G$-completeness is replaced by $M$-completeness. 
Authors' contributions

YS carried out the main proofs and drafted the manuscript. WC provided some valuable suggestions and helped to modify the manuscript. All authors read and approved the final manuscript.

\section{Author details}

${ }^{1}$ School of Mathematics and Statistics, Tianshui Normal University, Tianshui, 741001, P.R. China. ${ }^{2}$ School of Mathematics, Beijing Institute of Technology, Beijing, 100081, P.R. China. ${ }^{3}$ School of Information, Capital University of Economics and Business, Beijing, 100070, P.R. China.

\section{Acknowledgements}

This work was supported by 'Qing Lan' Talent Engineering Funds by Tianshui Normal University. The second author acknowledges the support of the Beijing Municipal Education Commission Foundation of China (No. KM201210038001), the Humanity and Social Science Youth Foundation of Ministry of Education of China (No. 13YJC630012).

Received: 30 September 2012 Accepted: 3 May 2013 Published: 20 May 2013

\section{References}

1. Grabiec, M: Fixed points in fuzzy metric spaces. Fuzzy Sets Syst. 27, 385-389 (1988)

2. Mishra, SN, Sharma, N, Singh, SL: Common fixed points of maps on fuzzy metric spaces. Int. J. Math. Math. Sci. 17, 253-258 (1994)

3. Vasuki, R: A common fixed point theorem in a fuzzy metric space. Fuzzy Sets Syst. 97, 395-397 (1998)

4. Cho, YJ: Fixed points in fuzzy metric spaces. J. Fuzzy Math. 5(4), 949-962 (1997)

5. Singh, B, Chauhan, MS: Common fixed points of compatible maps in fuzzy metric spaces. Fuzzy Sets Syst. 115 , 471-475 (2000)

6. Sharma, S: Common fixed point theorems in fuzzy metric spaces. Fuzzy Sets Syst. 127, 345-352 (2002)

7. Gregori, V, Sapena, A: On fixed point theorems in fuzzy metric spaces. Fuzzy Sets Syst. 125, 245-253 (2002)

8. Mihet, D: A Banach contraction theorem in fuzzy metric spaces. Fuzzy Sets Syst. 144, 431-439 (2004)

9. Mihet, D: On fuzzy contractive mappings in fuzzy metric spaces. Fuzzy Sets Syst. 158, 915-921 (2007)

10. Mihet, D: A class of contractions in fuzzy metrics paces. Fuzzy Sets Syst. 161, 1131-1137 (2010)

11. Abbas, M, Imdad, M, Gopal, D: $\varphi$-Weak contractions in fuzzy metric spaces. Iran. J. Fuzzy Syst. 8, 141-148 (2011)

12. Shen, YH, Qiu, D, Chen, W: Fixed point theorems in fuzzy metric spaces. Appl. Math. Lett. 25, 138-141 (2012)

13. Kirk, WA, Srinivasan, PS, Veeramani, P: Fixed points for mappings satisfying cyclical contractive conditions. Fixed Point Theory 4, 79-89 (2003)

14. Păcurar, M, Rus, IA: Fixed point theory for $\varphi$-contractions. Nonlinear Anal. 72, 1181-1187 (2010)

15. Karapinar, E: Fixed point theory for cyclic weak $\phi$-contraction. Appl. Math. Lett. 24, 822-825 (2011)

16. Shen, YH, Qiu, D, Chen, W: Fixed point theory for cyclic $\varphi$-contractions in fuzzy metric spaces. Iran. J. Fuzzy Syst. (2013, to appear)

17. Gopal, D, Imdad, M, Vetro, C, Hasan, M: Fixed point theory for cyclic weak $\phi$-contraction in fuzzy metric spaces. J. Nonlinear Anal. Appl. (2012). doi:10.5899/2012/jnaa-00110

18. Murthy, PP, Mishra, U, Rashmi, Vetro, C: Generalized $(\varphi, \psi)$-weak contractions involving $(f, g)$-reciprocally continuous maps in fuzzy metric spaces. Ann. Fuzzy Math. Inf. 5(1), 45-57 (2013)

19. Schweizer, B, Sklar, A: Statistical metric spaces. Pac. J. Math. 10, 385-389 (1960)

20. George, A, Veeramani, P: On some results in fuzzy metric spaces. Fuzzy Sets Syst. 64, 395-399 (1994)

21. Vetro, C: Fixed points in weak non-Archimedean fuzzy metric spaces. Fuzzy Sets Syst. 162, 84-90 (2011)

doi:10.1186/1687-1812-2013-133

Cite this article as: Shen and Chen: Fixed point theorems for cyclic contraction mappings in fuzzy metric spaces. Fixed Point Theory and Applications 2013 2013:133.

\section{Submit your manuscript to a SpringerOpen ${ }^{\circ}$ journal and benefit from:}

- Convenient online submission

- Rigorous peer review

- Immediate publication on acceptance

- Open access: articles freely available online

- High visibility within the field

- Retaining the copyright to your article 